ENSAYOS

\title{
Desafíos multidimensionales en la educación para migrantes en México
}

\author{
Multidimensional challenges in education \\ for migrants in Mexico
}

\author{
Norma Baca Tavira ${ }^{a}$ Silvia García Fajardo ${ }^{a}$, Maria Viridiana Sosa Márquez ${ }^{a}$ \\ ${ }^{a}$ Universidad Autónoma del Estado de México. \\ nbacat@uaemex.mx,sgarciaf@uaemex.mx,mvsosam@uaemex.mx
}

\section{RESUMEN}

El artículo presenta un panorama general de los desafíos en materia de política pública que México enfrenta en el contexto de la migración de retorno de Estados Unidos. Se analizan los factores sociales e institucionales que están dificultando el proceso de integración de menores, adolescentes y jóvenes migrantes a las comunidades escolares en los lugares de llegada. Se recupera información sobre acciones que el Estado mexicano ha desarrollado para atender a esta población y se reflexiona sobre los principales desafíos en la temática. Para desarrollar este trabajo se analizó información documental sobre la migración de retorno, programas gubernamentales de atención a la población migrante para su acceso al sistema educativo

Palabras claves: migración de retorno, educación y migración, desafíos, México.

\section{ABSTRACT}

The article presents an overview of the public policy challenges that Mexico faces in the context of return migration from the United States. The social and institutional factors that are hindering the process of integration of children, adolescents and young migrants to school communities in the places of arrival are analyzed. Information is retrieved from government actions that Mexico has developed to serve the population and ponder about the major challenges in the subject. In order to develop this work, documentary information on return migration, government programs for the attention of the migrant population for their access to the educational system was analyzed.

Key words: return migration, education and migration, challenges, Mexico. 


\section{INTRODUCCIÓN}

Durante el siglo XX, especialmente en la segunda mitad, las migraciones marcaron significativamente la dinámica sociodemográfica de México. Por un lado, el traslado de la población rural hacia áreas urbanas dio origen a un patrón de asentamiento con alta concentración espacial a tal punto que actualmente la migración y la movilidad cotidiana ejercen una fuerte influencia en la expansión territorial de las grandes ciudades. Contemporáneamente, la migración internacional, especialmente a Estados Unidos, fue constituyéndose de apoco en el mayor corredor migratorio del planeta. En 2015, según la Organización Internacional para las Migraciones (OIM, 2018, p. 35), México se ubicó como el segundo mayor país de origen y Estados Unidos el principal receptor, posición que ocupa desde 1970.

Sin duda alguna este sistema migratorio es uno de los de mayor tradición histórica y dinamismo en el mundo (Delgado y Márquez, 2007). La migración mexicana a Estados Unidos es considerada como una de las más importantes en la historia de la migración internacional por su complejidad en términos de su historicidad, desigualdad económica entre países, cantidad de población que circula. Para 2018, se estima una población de 12.8 millones de personas nacidas en México residiendo en el país vecino. Datos de la Encuesta Continua de Población (CPS) de Estados Unidos indican que la población de origen mexicana de segunda y tercera generación alcanzó los 38.4 millones en 2018. Es una enorme comunidad que se construyó durante décadas e influenciada principalmente por factores socioeconómicos y de desigualdades territoriales, de un lado, la oportunidad de acceder a mejores salarios y, por otro lado, la oportunidad de disponer de fuerza de trabajo barata y dócil a partir de su estatus migratorio.

Pero los trabajadores son personas, en su mayoría hombres jóvenes, que una vez instalados y con empleo buscaron establecerse en familia, llevar a sus esposas e hijos o bien unirse con mujeres migrantes y formar familias. También ha sido frecuente la presencia de familia extensa, como resultado de un proceso de reunificación familiar en Estados Unidos. En estas circunstancias es obvia la presencia de miembros de diferentes edades, con distintas necesidades en materia de empleo, salud y desde luego servicios educativos.

En espacios de movilidad transnacional la frecuencia e intensidad con la que la circulación migratoria se ha presentado han ido constituyendo formas de vida y de relaciones humanas que reproducen la continua presencia del fenómeno migratorio entre sus comunidades (Baca, 2011). Las comunidades transnacionales tienen su referente en el papel interactivo de las migraciones, por lo que destacan por su capacidad de establecer nuevas configuraciones culturales que difícilmente podrían emanar de un solo referente territorial. Con el desplazamiento espacial de la población se da el tránsito de identidades nacionales de base territorial a otras de índole transterritorial. Como se ha dicho, durante décadas en México miles de comunidades crearon diversos e intensos vínculos con miles de localidades en Estados Unidos porque las migraciones y la movilidad territorial representan un fenómeno cultural, político, económico, demográfico, social y psicológico de los grupos sociales y de la identidad individual.

Las comunidades transnacionales contribuyen a entender no sólo las modalidades y problemas de las migraciones y las movilidades contemporáneas en sus diferentes dimensiones, también permiten entender los cambios en las culturas nacionales y en los sistemas sociales y políticos, especialmente, en condiciones políticas, económicas y 
sociales cambiantes. Y cuando el gobierno estadunidense decidió endurecer la vigilancia y el control de la frontera con México, obviamente las condiciones de circulación de migrantes cambiaron. El patrón migratorio experimentó ajustes en su composición sociodemográfica, en las lógicas de circulación de las personas.

Las consecuencias de la política antiinmigrante se expresa en hechos como la disminución del flujo anual de migrantes no regulares debido entre otros factores al aumento en los riesgos del cruce y en el "costo de transacción" para cruzar la frontera de manera indocumentada, el pago promedio al pollero por parte de la población migrante mexicana para entrar a Estados Unidos se ha incrementado sorprendentemente, al pasar de 993 pesos en 1992 (aproximadamente 50 dólares actuales) a 87 mil en 2017 (aproximadamente 4,350 dólares actuales); también está el aumento en el número de aprehensiones en la frontera y desde el interior del territorio estadunidense. Durante el período 2007-2017, según el Departamento de Seguridad Interna de Estados Unidos, las autoridades de ese país realizaron más de 5.5 millones de aprensiones a nacionales mexicanos, 2008 registró el mayor número de aprehensiones a población mexicana por parte de las autoridades estadounidenses (884 mil). Aunado a lo anterior, el panorama económico en Estados Unidos se complicó pues esta nación registró recesión económica afectando directamente a la población trabajadora migrante, sin duda un escenario complicado para los migrantes indocumentados, especialmente para aquellos que habían formado familia en este destino. Zúñiga (2013, p. 5) explica que la existencia de vida familiar de los migrantes en Estados Unidos da cuenta del crecimiento sostenido de la matrícula de alumnos latinos, en su mayoría mexicanos, en el sistema escolar de Estados Unidos en la década de los noventa y principios de 2000.

Por ello, para el periodo 2008-2015, en pleno proceso de retorno de mexicanos, voluntario o involuntario (por deportación) a México, un punto sensible en las comunidades de migrantes es lo relativo a la integración social de los miembros de la familia, para algunos de ellos significa reconectarse con relaciones sociales conocidas pero para otros, para los menores, adolescentes y jóvenes, descubrir y entender las formas de convivencia en estos lugares y desarrollar estrategias para su adaptación a la comunidad representan retos por atender.

Así, la situación que planteamos en este artículo es de que a partir de 2005, miles de niños, adolescentes y jóvenes mexicanos que vivían y estudiaban en Estados Unidos comenzaron a regresar o llegaron por primera vez a México y son inscritos en escuelas del país. Bajo este contexto este trabajo se propone presentar algunos elementos que den cuenta de la dimensión y características de la relación migración-educación para el caso de México con énfasis en la migración de retorno.

Se trata de un estudio exploratorio que a partir de revisión documental analiza el estado de la cuestión en relación con los desafíos que la sociedad mexicana está enfrentando en el marco de la actual fase migratoria caracterizada por el retorno de importantes volúmenes de población desde Estados Unidos. La dimensión de la migración de retorno que se estudia es la de demanda de servicios educativos. El trabajo se integra de cuatro partes, en la introducción se acota espacial y temporalmente la problemática, mientras que en la segunda parte nos ocupamos de presentar sintéticamente las fases de la constitución del sistema migratorio México-Estados Unidos así como identificar elementos de cambio y de continuidades en esta dinámica migratorio internacional y abordar las problemáticas en materia educativa. En la tercera se presenta el panorama de la migración de retorno para 
el caso de México e identificar más específicamente los desafíos en la relación migracióneducación, cerramos con un apartado de reflexiones finales.

\section{MIGRACIÓN EN MÉXICO. CAMBIOS, CONTINUIDADES Y DESAFÍOS}

La historia de la migración México-Estados Unidos se caracteriza por una serie de periodos durante los cuales los patrones migratorios se transforman y evolucionan como respuesta a los cambios en la política migratoria de Estados Unidos. A partir de los años cuarenta la historia de este proceso puede ser dividida en al menos tres etapas. En términos generales la primera se identifica durante la operación del programa Bracero (1942 y 1964); un segundo momento comprende la finalización de dicho programa y el inicio de un importante flujo de migración no documentada, especialmente en los años 80; el tercer periodo está marcado por la intensificación de la vigilancia fronteriza a partir de la segunda mitad de los ochenta (Bautista y Rodríguez, 2017; Tuirán y Ávila, 2010). Durante medio siglo se desarrolló una circularidad migratoria importante entre ambos países, que permitió la acumulación de capital social migratorio y el desarrollo de comunidades transnacionales.

Es en la década de 1990 que se presenta un punto de inflexión para la circularidad de personas migrantes entre México y Estados Unidos; de acuerdo con Massey, Pren y Durand $\left(2009\right.$, p. 101) con la aplicación de políticas antiinmigrantes (como IIRIRA ${ }^{1}$ y $\mathrm{AEDPA}^{2}$ ) se transitó a la 'era de la marginalización'. Desde entonces en Estados Unidos las penas para los indocumentados se han incrementado, subió el número de delitos por los cuales los inmigrantes podrían ser deportados, es decir, la tendencia hacia la criminalización de la migración no documentada es mayor (Donato y Armenta, 2011; Bautista y Rodríguez, 2017), aunado a lo anterior, los costos y riesgos al cruzar la frontera se incrementan y con esto la decisión de permanecer en territorio estadounidense, lo que significó un impacto profundo en el sistema migratorio mexicano que pasó de ser una migración de tipo circular, masculina y regional, a una migración establecida, familiar y de dimensiones nacionales, tanto en el país de origen como en el de destino, la aplicación de políticas migratorias restrictivas tuvo un impacto directo en las tasas de retorno (Massey, Pren y Durand, 2009, p. 101).

Para principios de la década del 2010, el sistema migratorio México-Estados Unidos registraba una nueva transición. Por un lado, el estimado de población mexicana en Estados Unidos era de 12 millones, por otro lado, según el Departamento de Seguridad Interna (DHS) de Estados Unidos en ese año hubo 632 mil aprensiones de mexicanos por autoridades de aquel país. Tanto la proliferación de leyes estatales anti-inmigrantes, especialmente dirigidas hacia los mexicanos, el incremento sin precedentes de las expulsiones ocurridas desde el interior del territorio estadunidense que afectaron a las familias de los indocumentados la crisis financiera y económica de 2008 en Estados Unidos, afectaron negativamente las oportunidades de empleo en los mercados de trabajo en los que tradicionalmente laboran los inmigrantes mexicanos en aquel país lo que da contexto a la transición migratoria, ahora el retorno (voluntario y por deportación) era una creciente realidad.

IIRIRA: Illegal Immigration Reform and Immigrant Responsibility Act.

AEDPA: Antiterrorism and Effective Death Penalty Act. 
Así, la década pasada se caracterizó por desaceleración de la migración, crecimiento del retorno y de las deportaciones desde Estados Unidos. Los cambios en los patrones migratorios, en la modificación de las trayectorias migratorias y en los planes de los migrantes en cuanto a estadía y formación de familia en Estados Unidos se afectaron ante la deportación, para aquellos que ya habían formado una familia en el lugar de destino, las afectaciones claramente se multiplican por cada persona que integra el grupo doméstico. Hay metas truncadas y retornos no planeados, ser devuelto a México se convierte en un problema muy profundo para los migrantes y sus familias (Mojica, 2018), los proyectos de trabajo de los adultos y las trayectorias educativas de jóvenes, adolescentes y niños se fracturan.

En 18 años, a partir de la década pasada, la población migrante que llega desde Estados Unidos a México se ha incrementado, el retorno es tanto de personas nacidas en México como por personas nacidas en Estados Unidos. Teniendo en cuenta que unos y otros migrantes forman parte de un mismo flujo, en el estudio de Bautista y Giorguli (2019, p. 14) se contabiliza como migración de retorno a las dos poblaciones; en esta estimación cuentan a los menores de 17 años nacidos en Estados Unidos que viven con un jefe de familia de nacionalidad mexicana; las autoras consideran que esta inclusión da un soporte amplio al estudio del retorno porque "permite analizar las problemáticas conjuntas de integración en su llegada al país". Teniendo en cuenta esto, se estima que al año 2015, 515,339 personas que cinco años atrás vivían en Estados Unidos habían retornado a México, esa cantidad estaría distribuida desigualmente entre las entidades que comprenden el territorio mexicano. En 2015, los cinco estados que recibieron más migrantes fueron Jalisco $(47,517)$, Michoacán (41,101), Baja California $(37,786)$, Guanajuato $(33,345)$ y el Estado de México $(32,344)$ (Bautista y Giorguli, 2019, p. 14). El retorno, es identificado como una nueva condición en el país en materia migratoria, porque hasta la década de 1990 este territorio nacional era considerado, por su volumen, como de emigrantes internacionales ${ }^{3}$, desde mediados de la década pasada, la creciente población que llegaba al país procedente de Estados Unidos planteaba un desafío para la estructura económica, social e institucional nacional y desde luego en el ámbito local pues había que responder con soluciones para la reintegración de los migrantes retornados.

Adicionalmente, y por diversas causas se han registrado cambios muy importantes en las dinámicas migratorias en América Latina, donde flujos que antes iban en un sentido se han revertido o han cambiado de destino, diversos países se han convertido en receptores de personas migrantes. Como regiones de expulsión sobresalen tres territorios: el triángulo norte de Centro América (Guatemala, Honduras y El Salvador), en el Caribe, Haití y en el caso de América del Sur, sin duda Venezuela. En todos los casos, hay contexto de crisis, lo que significa que las migraciones se están dando de manera más forzada que por un proyecto laboral, de reunificación familiar o escolar. En el caso de México, la población nacida en El Salvador, Guatemala y Honduras pasó de 49,430 en 1990 a 68,272 en 2015, siendo entre 2010 y 2015 el periodo en el que más creció (7.7\% en promedio cada año). Los hondureños son los migrantes que más han incrementado su presencia, aunque las personas de Guatemala son la mayoría de centroamericanos en territorio mexicano. Según la Red de Documentación de las Organizaciones Defensoras de Migrantes (Redodem), en 2018 había más de 36 mil personas en situación de movilidad provenientes de otras

En el año 2000, en Estados Unidos había poco más de nueve millones de personas nacidas en México, a este número había que sumar a los descendientes nacidos en el lugar de destino. 
naciones. De los provenientes de Centroamérica particularmente de Honduras, $28.6 \%$ tiene como objetivo asentarse en México. En el informe Procesos migratorios en México. Nuevos rostros, mismas dinámicas 2018 (ITESO, 2018) la Redodem plantea que "en 2018 se hicieron 30 mil solicitudes de refugio, 236\% más que 2016 y de ellas $42 \%$ iniciaron su trámite en el último trimestre del mismo año, que coincide con la llegada de las caravanas masivas que generaron la crisis migratoria".

Lo que tenemos entonces en materia de migración internacional en México es que socialmente enfrentamos diversos retos: migración de diferentes tipos, entre ellas la circular, migración de retorno, de tránsito, inmigración y refugio. Los perfiles de estas migraciones son distintos, predominando la migración familiar (a diferencia de décadas anteriores donde los migrantes eran básicamente hombres solteros), esta situación significa que las personas migrantes tienen diferentes necesidades, trabajo desde luego pero también de diversos servicios como salud, vivienda y educación, eso sin considerar lo relacionado con la salud emocional a la que básicamente se ha ignorado.

Bajo estas circunstancias, México se ha visto rebasado por la inmigración, no éramos receptores de migración, si bien las leyes se han mejorado en materia migratoria y tienen en cuenta la perspectiva de Derechos Humanos, administrativamente las instituciones no están suficientemente preparadas para la atención a la diversidad de personas migrantes, y en términos presupuestales también hay carencias para gestionar los flujos migratorios.

En este trabajo, nos concentramos en reflexionar en torno de las complejidades y de los desafíos que México enfrenta en cuanto a los servicios de educación para la población migrante, poniendo especial atención en la población en retorno porque para el sistema educativo del país significa una presión urgente de atender, siendo un flujo que se acumula y se mantiene con números elevados. Según el Consejo Nacional de Población, en el quinquenio 2005-2010 el flujo de retornados a México se incrementó en 200\% en comparación con el quinquenio anterior. El número de retornados de 2010 a 2015 alcanzó la cifra de 1,440, 000 personas (BBVA y Conapo, 2016). Datos más recientes indican que de diciembre de 2018 a noviembre de 2019, se registraron 207,741 personas mexicanas repatriadas de Estados Unidos y Canadá, "las personas repatriadas menores de edad suman 12,061, en tanto que 195,680 son mayores de 18 años" (INM, 2019, p. 1).

La familia migrante se sitúa como el nuevo elemento presente en el análisis del fenómeno migratorio del retorno en México, se trata de familias cuyos miembros suelen tener estatus migratorios mixtos, incluye a miembros nacidos en Estados Unidos lo que les confiere un carácter transnacional; se trata de personas biculturales que crecieron en un contexto social, económico y cultural diferente al que llegan a insertarse en las localidades del país por lo que el proceso de incorporación de los jóvenes y niños que llegan a residir en los lugares de origen de sus padres es complejo.

La migración de retorno ha sido considerada como el desplazamiento que implica volver a establecerse y permanecer en el lugar de origen (país, estado o localidad), también puede ser el fin del ciclo migratorio. Desde la perspectiva transnacional, se plantea que la migración de retorno es una forma de movilidad compleja y que existen varias modalidades de regresar, así como nexos entre el retorno definitivo y la visita de regreso (Hirai, 2013). En el presente trabajo se entiende por retornados a aquellas personas o familias que después de haber vivido una experiencia migratoria fuera de México regresan a través de mecanismos como la repatriación, deportación o de manera voluntaria a establecerse en México (Moctezuma, 2013). 


\section{PANORAMA DEL RETORNO Y LOS DESAFÍOS EN LA RELACIÓN MIGRACIÓN-EDUCACIÓN}

En su Radiografía de la migración de retorno 2015, Bautista y Giorguli (2019, p. 16) estiman que el grupo de edad de 5 a 14 años muestra un volumen importante en la composición por edades, $13.6 \%$ en los niños y $25.6 \%$ en las niñas, la mayor concentración de población retornada se encuentra en el grupo 15-39 años (53.9\% en los hombres y $45.5 \%$ en las mujeres). Teniendo en cuenta la edad a la que retornan los migrantes es más fácil comprender las necesidades que estas personas requieren en cuanto a servicios, claramente son puestos de trabajo y servicios educativos para niños y jóvenes migrantes desde Estados Unidos. Las mismas autoras, identifican que los jóvenes migrantes de 15 a 18 años son los que muestran los porcentajes más bajos de asistencia escolar en esas edades (67.7 \%), 5.5 puntos porcentuales por debajo del promedio nacional para ese grupo de edad (73.2 \%).

En general son menores, adolescentes y jóvenes que en su momento migraron con su papá, mamá o con ambos, una vez instalados en Estados Unidos y cuando hubo condiciones de asistir a la escuela fueron inscritos y debieron aprender inglés y acreditar materias de contenido académico en el idioma oficial de la Unión Americana. Después, al retorno de la familia a México son inscritos en escuelas mexicanas e inicia un nuevo proceso de adaptación, no solo académico sino social lo que desde luego pasa por la readaptación/ adaptación al lenguaje, lo que puede ser complejo según las condiciones en la que se presentó el retorno y el contexto de migración donde se viva (Jiménez, 2018). En este proceso son importantes las condiciones por las cuáles se dio el retorno y cuáles son las consecuencias. En general la adaptación de las personas retornadas se posibilita a partir de conocer las estructuras de la sociedad donde ahora se radica.

En el retorno, para los adultos la prioridad es incorporarse a la actividad económica lo que significa relacionarse con diversas personas de la comunidad, interactuar con viejos o nuevos conocidos. En el caso de los menores, adolescentes y jóvenes, el espacio escolar vendría a ser central para la socialización y para el proceso de adaptación.

Son diversos los desafíos que se generan con la migración de retorno bajo las características descritas en el párrafo anterior, porque si los menores o los jóvenes cuentan con las injustas experiencias de acoso y discriminación eso tiene consecuencias en la capacidad de las familias y las unidades educativas porque hay factores a tener en cuenta en las adecuaciones que el sistema educativo debe hacer a efecto de prestar servicios escolares para una población diversa. Bajo este contexto, la política educativa en México debería ser flexible en diferentes ámbitos y niveles de escolaridad teniendo en cuenta la demanda de servicio de la población que reside en los territorios.

Por ejemplo, la demanda de escuela para los menores es diferente que para los jóvenes que necesitan educación superior, cuyas trayectorias académicas ya acumulan experiencia, han seguido una lógica de estructuración de los créditos y contenidos cursados, y por lo general son bilingües. Otra distinción a tener en cuenta es entre los niños nacidos en México que pasan por dos transiciones: escuela mexicana a escuela en Estados Unidos y luego escuela mexicana, a estas personas como alumnos transnacionales y los niños nacidos en Estados Unidos que cuentan con doble nacionalidad y, para quienes el tránsito es uno, se les identifica como estudiantes binacionales (Cortez, 2012; Zúñiga, 2013; Jiménez, 2018).

Estudios sobre estas poblaciones en México mayormente estudiadas en el norte y el occidente del país, por la mayor presencia de retornados como los que forman parte del 
texto Movilización, migración y retorno de la niñez migrante. Una mirada antropológica, identifican que la población que llega a Estados Unidos a cursar la educación básica presentan antecedentes de segregación e incluso señalamientos como criminales por el estatus de migrantes indocumentados. Estos menores han pasado incluso por revisión de su documentación, en tanto leyes como la HB56 en el estado de Alabama, mandata a las escuelas revisar el estado migratorio de sus estudiantes y actas de nacimiento para comprobar su nacionalidad; bajo este respaldo jurídico, "las autoridades estatales hostigan a los padres de familia y a los menores durante su camino a las escuelas, con el objetivo de solicitar sus papeles y deportarlos" (Ruíz y García, 2015, p. 305). Contar con experiencias de hostilidad como la descrita, impacta en el perfil del estudiante migrante de retorno, por ejemplo, en su sensibilidad hacia los espacios escolares, en su autoestima y en sus procesos de socialización.

En México los procesos administrativos que los migrantes de retorno deben cubrir para ingresar al sistema educativo llegan a excederse burocráticamente e incluso hay requisitos que resultan obsoletos, en tanto no hay correspondencia entre la solicitud de requerimientos y lo que establece el derecho a la educación, es decir el artículo 3ro constitucional, que mandata privilegiar la atención educativa en el país sin importar la condición migratoria de la persona tal como cita el artículo 8vo de la Ley de Migración (Cámara de Diputados, 2019). Siendo así, los migrantes provenientes de Estados Unidos son sujetos de inclusión escolar, no obstante, es frecuente que las personas migrantes hayan sido excluidas, de hecho, como plantea Eunice Vargas (2019, p. 28) "por la falta de estrategias eficientes para la equidad en el acceso a una escuela y a las oportunidades de aprendizaje" otros migrantes están en proceso de exclusión educativa. Se trata de procesos que dificultan la incorporación y continuidad en los estudios de la población migrante.

Lo anterior, no obstante que México cuenta con una robusta legislación y marco de políticas públicas de atención a migrantes. Derechos reconocidos por la Constitución Política de los Estados Unidos Mexicanos, las leyes de migración y población y las reglamentaciones específicas sobre la condición de nacionalidad. Adicionalmente, la nación se ha caracterizado por contar con una política migratoria que ha permitido que muchos extranjeros hayan encontrado una vida en este territorio, lo cual se remonta a la época de las dictaduras y exilios tanto en Latinoamérica como en Europa, aunado a las corrientes migratorias centroamericanas y suramericanas que en la última década imponen nuevos retos a la sociedad e institucionalidad de este país (Olvera, 2017, p. 33).

De acuerdo con el Artículo 2 de la Ley de Migración (2019, p. 2), la política migratoria deberá basarse en los siguientes principios:

Respeto irrestricto de los derechos humanos de los migrantes, nacionales y extranjeros, sea cual fuere su origen, nacionalidad, género, etnia, edad y situación migratoria, con especial atención a grupos vulnerables como menores de edad, mujeres, indígenas, adolescentes y personas de la tercera edad, así como a víctimas del delito.

En ningún caso una situación migratoria irregular preconfigurará por sí misma la comisión de un delito ni se prejuzgará la comisión de ilícitos por parte de un migrante por el hecho de encontrarse en condición no documentada.

Congruencia de manera que el Estado mexicano garantice la vigencia de los derechos que reclama para sus connacionales en el exterior, en la admisión, ingreso, permanencia, tránsito, deportación y retorno asistido de extranjeros en su territorio. 
Enfoque integral acorde con la complejidad de la movilidad internacional de personas, que atienda las diversas manifestaciones de migración en México como país de origen, tránsito, destino y retorno de migrantes, considerando sus causas estructurales y sus consecuencias inmediatas y futuras.

Responsabilidad compartida con los gobiernos de los diversos países y entre las instituciones nacionales y extranjeras involucradas en el tema migratorio.

Hospitalidad y solidaridad internacional con las personas que necesitan un nuevo lugar de residencia temporal o permanente debido a condiciones extremas en su país de origen que ponen en riesgo su vida o su convivencia, de acuerdo con la tradición mexicana en este sentido, los tratados y el derecho internacional.

Facilitación de la movilidad internacional de personas, salvaguardando el orden y la seguridad. Este principio reconoce el aporte de los migrantes a las sociedades de origen y destino. Al mismo tiempo, pugna por fortalecer la contribución de la autoridad migratoria a la seguridad pública y fronteriza, a la seguridad regional y al combate contra el crimen organizado, especialmente en el combate al tráfico o secuestro de migrantes, y a la trata de personas en todas sus modalidades.

Complementariedad de los mercados laborales con los países de la región, como fundamento para una gestión adecuada de la migración laboral acorde a las necesidades nacionales.

Equidad entre nacionales y extranjeros, como indica la Constitución Política de los Estados Unidos Mexicanos, especialmente en lo que respecta a la plena observancia de las garantías individuales, tanto para nacionales como para extranjeros.

Reconocimiento a los derechos adquiridos de los inmigrantes, en tanto que los extranjeros con arraigo o vínculos familiares, laborales o de negocios en México han generado una serie de derechos y compromisos a partir de su convivencia cotidiana en el país, aún cuando puedan haber incurrido en una situación migratoria irregular por aspectos administrativos y siempre que el extranjero haya cumplido con las leyes aplicables.

Unidad familiar e interés superior de la niña, niño y adolescente, como criterio prioritario de internación y estancia de extranjeros para la residencia temporal o permanente en México, junto con las necesidades laborales y las causas humanitarias, en tanto que la unidad familiar es un elemento sustantivo para la conformación de un sano y productivo tejido social de las comunidades de extranjeros en el país. Integración social y cultural entre nacionales y extranjeros residentes en el país con base en el multiculturalismo y la libertad de elección y el pleno respeto de las culturas y costumbres de sus comunidades de origen, siempre que no contravengan las leyes del país.

Facilitar el retorno al territorio nacional y la reinserción social de los emigrantes mexicanos y sus familias, a través de programas interinstitucionales y de reforzar los vínculos entre las comunidades de origen y destino de la emigración mexicana, en provecho del bienestar familiar y del desarrollo regional y nacional (Cámara de Diputados, 2019, p. 2).

Además, el Programa Especial de Migración (PEM), 2014-2018, es valorado como un avance loable en la definición de una política migratoria acorde con la complejidad del fenómeno migratorio en México, pues entre sus objetivos sobresale el fomento de una cultura de derechos humanos, el impulso al desarrollo regional y local, la promoción de 
una gestión migratoria con corresponsabilidad, la integración laboral, cultural y política de la población migrante y el fortalecimiento de su acceso al sistema de justicia (Segob, 2014). En la actual administración federal, el Gobierno de México anuncia que la nueva política migratoria 2018-2024:

impulsa un cambio de modelo para la atención del tema migratorio en sus cuatro dimensiones: origen, tránsito, destino y retorno; donde contempla la movilidad humana segura, ordenada y regular; colocando al centro a las personas migrantes, al desarrollo social, cultural y económico. Según la Secretaría de Gobernación, bajo esta propuesta se "reorienta la política en la materia con acciones que dignifican el trato hacia las personas migrantes y que reconocen sus contribuciones a las sociedades en las que se encuentran (Segob, 2019, s/p).

Como decíamos, a pesar de la existencia de un robusto sistema normativo de protección a los derechos de la población migrante, con relativa frecuencia en México se observan en la práctica múltiples y diversas situaciones de discriminación y violación a los derechos humanos de las personas migrantes. En esta línea de reconocimiento, estudiosos de la relación migración, educación y derechos humanos (García y Tarrío, 2008; García, 2015; Olvera, 2017) coinciden en la necesidad de poner atención a dos formas frecuentes de violencia contra los migrantes: la institucional y no institucional. En el caso de la institucional ocurre mediante la implementación de leyes y normativas no coincidentes con el marco de los derechos humanos y por prácticas de abuso que funcionarios públicos ejercen a partir del desconocimiento de garantías y derechos que la ley otorga a la población migrante, independientemente de su estatus legal. Como se sabe, el hecho de que haya desconocimiento de la ley no exime de responsabilidad a las autoridades, en todo caso correspondería implementar programas de capacitación al personal a fin de resolver esta carencia.

La violencia no institucional puede identificarse como la que ejerce la comunidad en las sociedades de destino, suele ser de tipo cultural, simbólica y física, por lo que incluye actos de discriminación por diversos rasgos: racial, de género, orientación sexual, edad, actividad económica o por origen territorial que denigran la dignidad humana de los migrantes.

En la articulación de ambos tipos de violencia debe entonces considerarse que la

obligación del Estado en materia de derechos humanos no se reduce a la responsabilidad de respetarlos, en el sentido de conformar los ordenamientos necesarios y verificar que sus agentes actúen de acuerdo con los parámetros de legalidad, sino también debe tutelar que sean efectivamente respetados por la sociedad (Garza, 2015, p. 233).

En todo caso, el panorama es complicado para el país y la urgencia por fortalecer las instituciones que atienden a la población migrante es cada vez mayor. En cuanto a las dificultades para acceder al sistema educativo y, en coincidencia con Vargas (2019) las barreras para la educación se clasifican en:

[...] estructurales, culturales y sociales. Destacan entre las barreras estructurales: la falta de acceso a escuelas, al supeditar la matriculación del estudiante transnacional a la disponibilidad de cupo o a la posesión de documentos de identidad o de los 
antecedentes escolares, aun cuando por normativa éstos no son requeridos. Entre las barreras culturales sobresalen: las dificultades con el manejo del español de los estudiantes transnacionales y el escaso bilingüismo del personal educativo, así como las diferencias socioculturales en los contextos escolares y los contenidos curriculares entre los sistemas estadounidenses y el mexicano. Y, por último, entre las barreras sociales resaltan: la separación familiar y la falta de conexiones sociales en las escuelas, así como la dispersión escolar de estos estudiantes (p. 28).

Sin duda las especificidades debido a la amplia diversidad de circunstancias en las que ocurre el retorno y en los que se vive la experiencia de acercamiento al sistema escolar en México dan una larga lista de problemáticas, en el desarrollo de este trabajo se han mencionado algunas, a las que agregamos la falta de espacios educativos, es decir, planteles y profesorado en las regiones del país. En las ciudades capitales de las entidades hay infraestructura escolar, pero suele estar saturada, no hay cupo lo que implica que deben esperar a un siguiente ciclo escolar para postularse o inscribirse lo que significa desfase en la trayectoria académica.

De acuerdo con la SEP (2015), a manera de resumen y, para cerrar este apartado, mencionamos cinco dificultades que se presentan específicamente en el proceso de integración de niños y adolescentes en el sistema educativo mexicano, los estudiantes se sienten invisibles como sujetos sociales porque existe muy escasa o nula evidencia de su origen educativo o su trayectoria académica binacional; estos estudiantes registran niveles de frustración considerables al no sentirse reconocidos en el aula ante ciertas fallas de desempeño por no poder completar sus tareas o leer o escribir, en general consideran que se ignora su fondo de conocimiento; los estudiantes piensan que el profesorado en México no responde a sus necesidades por la diferencia de idioma y cultural en ese mismo sentido, una cuarta dificultad es que consideran que el sistema educativo de Estados Unidos es superior porque les proporciona actividades extras que les permite desarrollar su potencial, y por último, viven con cambios constantes en cuanto a contenidos escolares, materiales y actividades, esta situación los conflictúa porque el proceso de adaptación social y cultural no está en un punto que les proporcione mayor respaldo para su desempeño académico.

\section{MIGRACIÓN Y POLÍTICAS PÚBLICAS. ATENDER LA COMPLEJIDAD}

Consideramos que México presenta avances en el reto de favorecer la incorporación de los migrantes en el sistema educativo del país. Por ejemplo, teniendo en cuenta que en todo México $10.9 \%$ de la población retornada ha sido registrada en otro país, y que $2.2 \%$ no cuenta con un acta de nacimiento (Bautista y Giorguli, 2019), unas dificultades recurrentes en el proceso de inscripción a las escuelas son el reconocimiento de la binacionalidad y contar con documentos de identidad, en ese sentido, una respuesta positiva del Estado, ha sido la eliminación de la apostilla y la traducción oficial por perito de los documentos emitidos en Estados Unidos para la inserción y la certificación en los niveles de educación básica y media superior (SEP, 2015).

Para dar orientación a los migrantes en materia educativa, la SEP y la Secretaría de Relaciones Exteriores (SER), a través del Instituto de los Mexicanos en el Exterior (IME), han puesto a disposición de la población migrante en México y en Estados Unidos 37 
Ventanillas de Orientación Educativa que son espacios en los consulados para difundir entre connacionales mexicanos las oportunidades educativas que existen en México y en Estados Unidos. También el IME, desarrolla en territorio estadunidense las llamadas Plazas comunitarias, cuyo objetivo es realizar actividades culturales y de fomento a la lectura con la comunidad de paisanos. De la misma forma que las ventanillas este programa funciona a través de los consulados quienes también organizan la semana binacional de educación, actividad que para su segunda emisión en 2018 reportó haber atendido a más de 160 mil personas en los 50 consulados con los que cuenta México en territorio estadunidense. Respaldado por la SRE también tiene un fondo de becas para estudiantes transnacionales.

Un programa para estudiantes mexicanos que se encuentran residiendo en Estados Unidos es el de Intercambio de Maestros México-Estados Unidos en el que hasta 2015 habían participado más de 3,200 profesores. Se trata de una estrategia de cooperación internacional de alta pertinencia educativa para atender la demanda de maestros bilingües en distritos escolares en Estados Unidos que reciben a niños de origen mexicano que no dominan el idioma inglés. A su regreso los docentes replican en sus centros escolares, específicamente con población migrante, las habilidades desarrolladas.

Los maestros en intercambio son profesionales de la educación con experiencia como docente en diferentes niveles educativos, desde preescolar hasta profesional. Entre los participantes hay profesionales que o dominan el inglés o alguna lengua indígena de México, además se toma en consideración para ser elegibles el contar con conocimientos generales de historia, cultura, costumbres y tradiciones mexicanas.

Un programa que debe mencionarse por ser pionero (nace en 1982) y porque en lo general es reconocido como una acción positiva del Estado para fortalecer la colaboración entre los sistemas escolares estatales de México y Estados Unidos, lo lleva a cabo la Secretaría de Educación Pública (SEP), nos referimos al Programa Binacional de Educación Migrante (Probem). Este programa posibilita otorgar reconocimiento oficial a los estudios de educación básica que lleva a cabo la población migrante México-Estados Unidos y viceversa, acompañando el proceso de ubicación en la escuela de recepción, de acuerdo con su edad, y el grado académico correspondiente. En México, la educación es un derecho por lo que en caso de acudir a las delegaciones estatales o las instalaciones de la SEP a demandar el ejercicio de este derecho, la institución está obligada a respaldar y resolver la ubicación del estudiante solicitante en una escuela donde haya cupo.

\section{REFLEXIONES FINALES}

Las migraciones y las movilidades que se están presentando en la actualidad o la tendencia de estas movilidades humanas son hacia la diversidad de territorios vinculados por las personas migrantes. Migraciones de ida y vuelta, o solo de ida o con dirección opuesta. Hoy más que nunca, el fenómeno migratorio se presenta altamente complejo, especialmente para países como México, que no obstante su larga historia de migración hoy enfrenta retos mayúsculos no solo por el retorno de población mexicana desde Estados Unidos sino por la creciente migración de tránsito con tendencia a establecerse en territorio mexicano lo que significa proveer de servicios básicos a estas personas.

En este trabajo se han planteado las que consideramos son las principales barreras que, en materia escolar, los migrantes de retorno están enfrentando. De entre los desafíos, en 
específico institucionales, estamos convencidas que la mejora en la atención a los retornados implica una re-funcionalidad de los modelos educativos en los lugares de origen, destino y retorno, esto es, instaurar un sistema educativo que no sólo corresponda con los procesos de movilidad de las familias, en particular de sus hijos en edades escolares, sino que a su vez se flexibilice y se adapte en sus procesos administrativos y estructuras curriculares, con la finalidad de favorecer la interculturalidad, lograr una mejor intermediación del idioma y la inclusión e integración de la población migrante en su diversidad socioeconómica y cultural.

En materia de política pública, nos parece muy importante que las líneas de acción para la integración educativa de estos estudiantes deben involucrar a los actores de las escuelas, es decir, que pase del nivel o sector administrativo a la cotidianidad de las comunidades escolares en las diferentes regiones del país. Asimismo, debe capacitarse al funcionariado de los sistemas escolares federales y estatales para que cuenten con el conocimiento suficiente sobre las normativas que rigen a los asuntos migratorios y su relación con el sector educativo.

Somos conscientes que este ejercicio de exploración en la situación que prevalece en México sobre la relación migración educación, está muy lejos de cubrir en la relatoría, una porción mayoritaria de las diversas circunstancias en las que se presentan las migraciones entre México y Estados Unidos en torno del acceso al sistema escolar, el panorama es complejo, amplio y variado, por ejemplo, algunas de las problemáticas que no se tocaron en su especificidad son las que padecen los estudiantes transnacionales en el nivel superior, tampoco hablamos de migrantes circulares, que de forma cotidiana desarrollan su trayectoria migratoria entre los dos países, asimismo, está la situación de los estudiantes en México no han migrado nunca a Estados Unidos, pero que, sin embargo, están expuestos a la migración internacional por diversas circunstancias: su padre, su madre o sus hermanos (los miembros de su propia familia nuclear) están viviendo en ese país mientras ellos se encuentran estudiando en las escuelas mexicanas. México es un país con una amplísima variedad de comunidades transnacionales.

Por último, mencionamos dos retos más: problemas de coordinación entre entidades de diferente orden de gobierno y la necesidad de mayor presupuesto para operar y para ampliar la cobertura de servicios educativos en México para toda la población, porque la educación es un derecho.

\section{REFERENCIAS BIBLIOGRÁFICAS}

Baca, N. (2011). Lógicas de circulación y migración femenina del sur mexiquense a Estados Unidos. Ciudad de México: UNAM.

Bautista, A. y Giorguli, S. (2019). Radiografía de la migración de retorno 2015. En S. Giorguli et al. (coords.), Migración de retorno y derechos sociales. Barreras a la integración (pp. 13-26). Ciudad de México: Comisión Nacional de Derecho Humanos / El Colegio de México.

Bautista, A. y Rodríguez, M. (2017). Cambios y continuidades en el proceso migratorio de las mujeres del Estado de México a Estados Unidos. En J. Olvera y N. Baca (coords.), Continuidades y cambios en las migraciones de México a Estados Unidos (pp. 259-284). Toluca: Universidad Autónoma del Estado de México.

BBVA y Conapo (Fundación Bancomer y Consejo Nacional de Población). (2016). Anuario Estadístico de Migración y remesas, 2016. Ciudad de México: BBVA / Conapo. 
Cámara de Diputados. (2019). Ley de Migración. En Diario Oficial de la Federación, 3 de julio. Ciudad de México: Congreso de la Unión.

Cordero, M. (2017). Mujeres adolescentes y jóvenes, migración e inclusión educativa, Tesis de maestría en Ciencias Sociales con mención en Género y Desarrollo. Quito: Flacso Andes.

Cortez R., N. (2012). Bilingües y Biculturales. Estudiantes universitarios migrantes de retorno. En G. C. Valdez Gardea (coord.), Movilización, migración y retorno de la niñez migrante. Una mirada antropológica (pp. 215-221). Hermosillo: El Colegio de Sonora.

García, M. C. y Tarríos, M. (2008). Migración irregular centroamericana, las tensiones entre derechos humanos, ley y justicia. Tuxtla Gutiérrez: Miguel Ángel Porrúa.

Garza, M. T. (2015). El marco legal nacional e internacional sobre migración y los derechos humanos. En K. Andrade y S. P. Izcara (coords.) Migrantes, transmigrantes, deportados y derechos humanos. Un enfoque binacional (pp. 58-74). Ciudad de México: Fontamara.

Delgado, R. y Márquez, C. (2007). El sistema migratorio México-Estados Unidos: dilemas de la integración regional, el desarrollo y la migración. En S. Castles y R. Delgado (coords.), Migración y desarrollo: perspectivas desde el sur (pp. 5-19). Ciudad de México: Miguel Ángel Porrúa.

Donato, K. \& Armenta, A. (2011). What we know about unauthorized migration. In Annual Review of Sociology (pp. 529-543), (37), Washington D.C.: University of Washington.

Hirai, S. (2013). Formas de regresar al terruño en el transnacionalismo. Apuntes teóricos sobre migración de retorno. En Alteridades (pp. 95-105). Ciudad de México: Universidad Autónoma Metropolitana.

INM (Instituto Nacional de Migración). (2019). Boletín 381 del INM, 27 de diciembre, Ciudad de México: INM.

ITESO (Instituto Tecnológico y de Estudios Superiores de Occidente). (2018). Procesos migratorios en México. Nuevos rostros, mismas dinámicas 2018. Guadalajara: Redomem.

Jiménez, I. D. (2018). Jóvenes migrantes de retorno. Proceso de socialización en el contexto escolar. En I. García y E. Sánchez (coords.), Migraciones globales, redes sociales y construcción de espacio público de migrantes transnacionales (pp. 63-78), Xalapa: Red Iberoamericana de Academias de Investigación, A. C.

Massey, D., Pren, K. y Durand, J. (2009). Nuevos escenarios de la migración México-Estados Unidos. Las consecuencias de la guerra antiinmigrante. En Papeles de Población (pp. 101-128), (61), julio-septiembre, Toluca: Universidad Autónoma del Estado de México.

Mendoza, C. (2019). Jóvenes migrantes circulares entre Michoacán y California: narrativas y experiencia familiar, escolar e identitaria, 2006-2016 (228), (Tesis de doctorado en Ciencias Sociales). Zamora: El Colegio de Michoacán.

Moctezuma, M. (2013). Retorno de migrantes a México. Su reformulación conceptual. En Papeles de población (pp. 149-175), (77), Toluca: Universidad Autónoma del Estado de México.

Mojica, A. (2018). Migrantes deportados con retorno voluntario-forzado y algunos efectos recientes en los circuitos migratorios posteriores a la crisis de 2008. En N. Baca y A. Mojica (coords.) Movilidades y migrantes internacionales. Reflexiones sobre campos de relaciones socioeconómicas en comunidades de migrantes en México y Estados Unidos (pp. 161-178). Ciudad de México: Gedisa.

Olvera, J. (2017). Derechos humanos, educación y participación de la población migrante. En J. Olvera y N. Baca (coords.), Continuidades y cambios en las migraciones de México a Estados Unidos (pp. 25-43). Toluca: Universidad Autónoma del Estado de México.

OIM (Organización Internacional para las Migraciones). (2018). Informe sobre las migraciones en el mundo. Ginebra: OIM.

Ruiz, L. y E. García (2015). Reseña. Valdez, G. C. (coord.) Movilización, migración y retorno de la niñez migrante. Una mirada antropológica. En Región y sociedad (pp. 299-309), (63), Hermosillo: El Colegio de Sonora. 
Segob (Secretaría de Gobernación). (2014). Programa Especial de Migración 2014-2018, Ciudad de México: Gobierno de la República.

(2019). Política para la integración y protección de migrantes, Ciudad de México: Gobierno de México. Disponible en http://portales.segob.gob.mx/es/PoliticaMigratoria\#003.

Tuirán, R. y Ávila, J. L. (2010). La migración México-Estados Unidos, 1940-2010. En Los grandes problemas de México (pp. 163-167), Ciudad de México: El Colegio de México.

Vargas, E. (2019). Migración de retorno e integración educativa. En S. Giorguli et al. (coords.), Migración de retorno y derechos sociales. Barreras a la integración (pp. 27-44). Ciudad de México: Comisión Nacional de Derecho Humanos / El Colegio de México.

Zúñiga, V. (2013). Migrantes internacionales en las escuelas mexicanas: desafíos actuales y futuros en la política educativa. En Sinéctica. Revista electrónica de Educación (pp. 1-7), (40), Guadalajara: Instituto Tecnológico y de Estudios Superiores de Occidente. 
